\title{
Chaos suppression in the SU(2) Yang-Mills-Higgs system
}

\author{
Luca Salasnich円 \\ Dipartimento di Fisica "G. Galilei" dell'Università di Padova, \\ Via Marzolo 8, I 35131 Padova, Italy \\ Departamento de Fisica Atomica, Molecular y Nuclear \\ Facultad de Ciencias Fisicas, Universidad "Complutense" de Madrid, \\ Ciudad Universitaria, E 28040 Madrid, Spain
}

\begin{abstract}
We study the classical chaos-order transition in the spatially homogenous SU(2) Yang-Mills-Higgs system by using a quantal analog of Chirikov's resonance overlap criterion. We obtain an analytical estimation of the range of parameters for which there is chaos suppression.
\end{abstract}

PACS: 11.15.-q; 05.45.+b; 03.65.Sq

\footnotetext{
${ }^{1}$ Electronic address: salasnich@padova.infn.it
} 
In the last years there has been much interest in the chaotic behaviour of classical field theories. In particular have been studied Yang-Mills [1,2], Yang-Mills-Higgs [3,4,5], Abelian-Higgs [6], and also Chern-Simons [7] systems.

Usually the order-chaos transition in these systems has been studied numerically with Lyapunov exponents [8] and sections of Poincarè [9]. Less attention has been paid to analytical criteria. Some authors $[4,6]$ have used the curvature criterion of potential energy [10], but this criterion guarantees only a local instability and can give incorrect results (for a fuller discussion of this point see [11]).

In this work we study analytically the suppression of classical chaos in the spatially homogenous SU(2) Yang-Mills-Higgs (YMH) system. We apply a quantal analog [12] of the Chirikov resonance criterion [13] by using the semiclassical quantization to calculate the critical value of the parameters corresponding to the intersection of two neighboring quantal separatrices $[20]$.

Obviously, the constant field approximation implies that our SU(2) YMH system is a toy model for classical non-linear dynamics, with the attractive feature that the model emerges from particle physics.

The lagrangian density for the $\mathrm{SU}(2) \mathrm{YMH}$ system is given by [14]:

$$
L=-\frac{1}{4} F_{\mu \nu}^{a} F^{\mu \nu a}+\frac{1}{2}\left(D_{\mu} \phi\right)^{+}\left(D^{\mu} \phi\right)-V(\phi),
$$

where:

$$
\begin{gathered}
F_{\mu \nu}^{a}=\partial_{\mu} A_{\nu}^{a}-\partial_{\nu} A_{\mu}^{a}+g \epsilon^{a b c} A_{\mu}^{b} A_{\nu}^{c}, \\
\left(D_{\mu} \phi\right)=\partial_{\mu} \phi-i g A_{\mu}^{b} T^{b} \phi
\end{gathered}
$$


with $T^{b}=\sigma^{b} / 2, b=1,2,3$, generators of the $\mathrm{SU}(2)$ algebra, and where the potential of the scalar field (the Higgs field) is:

$$
V(\phi)=\mu^{2}|\phi|^{2}+\lambda|\phi|^{4}
$$

We work in the $(2+1)$-dimensional Minkowski space $(\mu=0,1,2)$ and choose spatially homogenous Yang-Mills and the Higgs fields:

$$
\partial_{i} A_{\mu}^{a}=\partial_{i} \phi=0, \quad i=1,2
$$

i.e. we consider the system in the region where space fluctuations of fields are negligible compared to their time fluctuations.

In the gauge $A_{0}^{a}=0$ and using the real triplet representation for the Higgs field we obtain:

$$
\begin{aligned}
L= & \frac{1}{2}\left({\dot{\overrightarrow{A_{1}}}}^{2}+{\dot{\overrightarrow{A_{2}}}}^{2}\right)+\dot{\vec{\phi}}^{2}-g^{2}\left[\frac{1}{2}{\overrightarrow{A_{1}}}^{2}{\overrightarrow{A_{2}^{2}}}^{2}-\frac{1}{2}\left(\overrightarrow{A_{1}} \cdot \overrightarrow{A_{2}}\right)^{2}+\right. \\
& \left.+\left(\vec{A}_{1}^{2}+\vec{A}_{2}^{2}\right) \vec{\phi}^{2}-\left(\overrightarrow{A_{1}} \cdot \vec{\phi}\right)^{2}-\left(\overrightarrow{A_{2}} \cdot \vec{\phi}\right)^{2}\right]-V(\vec{\phi}),
\end{aligned}
$$

where $\vec{\phi}=\left(\phi^{1}, \phi^{2}, \phi^{3}\right), \overrightarrow{A_{1}}=\left(A_{1}^{1}, A_{1}^{2}, A_{1}^{3}\right)$, and $\overrightarrow{A_{2}}=\left(A_{2}^{1}, A_{2}^{2}, A_{2}^{3}\right)$.

When $\mu^{2}>0$ the potential $V$ has a minimum in $|\vec{\phi}|=0$, but for $\mu^{2}<0$ the minimum is:

$$
\left|\vec{\phi}_{0}\right|=\left(\frac{-\mu^{2}}{4 \lambda}\right)^{\frac{1}{2}}=v
$$

which is the non zero Higgs vacuum. This vacuum is degenerate and after spontaneous symmetry breaking the physical vacuum can be chosen $\vec{\phi}_{0}=$ $(0,0, v)$. If $A_{1}^{1}=q_{1}, A_{2}^{2}=q_{2}$, and the other components of the Yang-Mills fields are zero, in the Higgs vacuum the hamiltonian of the system is:

$$
H=\frac{1}{2}\left(p_{1}^{2}+p_{2}^{2}\right)+g^{2} v^{2}\left(q_{1}^{2}+q_{2}^{2}\right)+\frac{1}{2} g^{2} q_{1}^{2} q_{2}^{2},
$$


where $p_{1}=\dot{q}_{1}$ and $p_{2}=\dot{q}_{2}$. Obviously $w^{2}=2 g^{2} v^{2}$ is the mass term of the Yang-Mills fields.

Classical chaos was demonstrated in a pure Yang-Mills system [1], i.e. in a zero Higgs vacuum. Here we analyze the effect of a non zero Higgs vacuum $[3]$.

We introduce the action-angle variables by the canonical transformation:

$$
q_{i}=\left(\frac{2 I_{i}}{\omega}\right)^{\frac{1}{2}} \cos \theta_{i}, \quad p_{i}=\left(2 I_{i} \omega\right)^{\frac{1}{2}} \sin \theta_{i}, \quad i=1,2 .
$$

The hamiltonian becomes (see also [3]):

$$
H=\left(I_{1}+I_{2}\right) \omega+\frac{1}{v^{2}} I_{1} I_{2} \cos ^{2} \theta_{1} \cos ^{2} \theta_{2}
$$

By the new canonical transformation in slow and fast variables:

$$
\begin{aligned}
& A_{1}=I_{1}+I_{2}, \quad A_{2}=I_{1}-I_{2}, \\
& \theta_{1}=\chi_{1}+\chi_{2}, \quad \theta_{2}=\chi_{1}-\chi_{2},
\end{aligned}
$$

$H$ can be written:

$$
H=A_{1} \omega+\frac{1}{4 v^{2}}\left(A_{1}^{2}-A_{2}^{2}\right) \cos ^{2}\left(\chi_{1}+\chi_{2}\right) \cos ^{2}\left(\chi_{1}-\chi_{2}\right) .
$$

We now eliminate the dependence on the angles to order $1 / v^{4}$ by resonant canonical perturbation theory [15]. First we average on the fast variable $\chi_{1}$. This yields:

$$
\frac{1}{2 \pi} \int_{0}^{2 \pi} d \chi_{1} \cos ^{2}\left(\chi_{1}+\chi_{2}\right) \cos ^{2}\left(\chi_{1}-\chi_{2}\right)=\frac{1}{8}\left(2+\cos 4 \chi_{2}\right)
$$

and:

$$
\bar{H}_{c l}=A_{1} \omega+\frac{1}{32 v^{2}}\left(A_{1}^{2}-A_{2}^{2}\right)\left(2+\cos 4 \chi_{2}\right) .
$$


The dependence on $\chi_{2}$ is now eliminated by a second canonical transformation. The Hamilton-Jacobi equation for the perturbation part is indeed:

$$
\begin{aligned}
& {\left[A_{1}^{2}-\left(\frac{\partial S}{\partial \chi_{2}}\right)^{2}\right]\left(2+\cos 4 \chi_{2}\right)=K,} \\
& \frac{\partial S}{\partial \chi_{2}}= \pm \sqrt{\frac{A_{1}^{2}\left(2+\cos 4 \chi_{2}\right)-K}{2+\cos 4 \chi_{2}}} .
\end{aligned}
$$

and thus the Hamiltonian becomes:

$$
\bar{H}=B_{1}+\frac{1}{32 v^{2}} K\left(B_{1}, B_{2}\right),
$$

where:

$$
B_{1}=A_{1}, \quad B_{2}=\frac{1}{2 \pi} \oint d \chi_{2} \frac{\partial S}{\partial \chi_{2}} .
$$

It appears from the structure of this equation that the motion of our system is similar that of a simple pendulum: for $0<K<B_{1}^{2}$ rotational motion, for $K=B_{1}^{2}$ separatrix, and for $B_{1}^{2}<K<3 B_{1}^{2}$ librational motion. On the separatrix we have $B_{1}^{2}\left(2+\cos 4 \chi_{2}\right)=K$, and:

$$
B_{2}= \pm \frac{2}{\pi} \int_{a}^{b} d x \sqrt{\frac{B_{1}^{2}(2+\cos 4 x)-K}{2+\cos 4 x}}
$$

where $a=-\frac{\pi}{4}, b=\frac{\pi}{4}$ for rotational motion, and $a=\phi_{-}\left(K, B_{1}\right), b=$ $\phi_{+}\left(K, B_{1}\right)$ for librational motion, with:

$$
\phi_{ \pm}\left(K, B_{1}\right)= \pm \frac{1}{4} \arccos \left(\frac{K}{B_{1}^{2}}-2\right) .
$$

The appearance of a separatrix (which is not immediately obvious in the $(p, q)$ coordinates) accounts as is well known for the stochastic layers originating near it [16]. This corresponds to local irregular behaviour of the quantum 
spectrum; one of its manifestations is the local shrinking of the level spacing and the tendency to avoided crossing $[16,17]$.

The approximate hamiltonian (16) depends only on the actions so that a semiclassical quantization formula can be obtained by the Bohr-Sommerfeld quantization rules $[15,18]$. Set $B_{1}=m_{1} \hbar$ and $B_{2}=m_{2} \hbar$, then, up to terms of order $\hbar$, the quantum spectrum is:

$$
E_{m_{1}, m_{2}}=m_{1} \hbar \omega+\frac{1}{32 v^{2}} K\left(m_{1} \hbar, m_{2} \hbar\right),
$$

where $K$ is implicitly defined by the relation:

$$
m_{2} \hbar= \pm \frac{2}{\pi} \int_{a}^{b} d x \sqrt{\frac{\left(m_{1} \hbar\right)^{2}(2+\cos 4 x)-K}{2+\cos 4 x}},
$$

with $a=-\frac{\pi}{4}, b=\frac{\pi}{4}$ for $0<K<\left(m_{1} \hbar\right)^{2}$, and $a=\phi_{-}\left(K, B_{1}\right), b=\phi_{+}\left(K, B_{1}\right)$ for $\left(m_{1} \hbar\right)^{2}<K<3\left(m_{1} \hbar\right)^{2}$.

On the separatrix, where $K=\left(m_{1} \hbar\right)^{2}, m_{2}= \pm \alpha m_{1}$, with:

$$
\alpha=\frac{2}{\pi} \int_{-\frac{\pi}{4}}^{\frac{\pi}{4}} d x \sqrt{\frac{1+\cos 4 x}{2+\cos 4 x}} .
$$

It is immediate to see that for $m_{1}$ fixed the function $K$, and hence the semiclassical energy $E_{m_{1}, m_{2}}$, is a decreasing function of the secondary quantum number $m_{2}$, and we have a quantum multiplet [19].

We can calculate the value of the coupling constant $1 / v^{2}$ corresponding to the intersection of the separatrices of two neighboring quantum multiplets:

$$
\left(m_{1}+1\right) \hbar \omega+\frac{1}{32 v^{2}} K\left[\left(m_{1}+1\right) \hbar, \alpha\left(m_{1}+1\right) \hbar\right]=m_{1} \hbar \omega+\frac{1}{32 v^{2}} K\left(m_{1} \hbar, \alpha m_{1} \hbar\right),
$$

and so:

$$
\frac{1}{v^{2}}=\frac{-32 \hbar \omega}{K\left[\left(m_{1}+1\right) \hbar, \alpha\left(m_{1}+1\right) \hbar\right]-K\left(m_{1} \hbar, \alpha m_{1} \hbar\right)} .
$$


In this way we have, in some sense, the quantal counterpart [12] of the method of overlapping resonances developed by Chirikov [13]. The denominator can be evaluated by the Taylor expansion and finally:

$$
\frac{1}{v^{2}}=\left[\frac{-8 \omega}{\frac{\partial K}{\partial B_{1}}-\alpha \frac{\partial K}{\partial B_{2}}}\right]_{B_{1}=m_{1} \hbar, B_{2}=\alpha m_{2} \hbar} .
$$

$\mathrm{K}$ is implicitly defined by the relation:

$$
F\left[B_{1}, B_{2}, K\left(B_{1}, B_{2}\right)\right]=B_{2}-\frac{\pi}{2} \int_{-\frac{\pi}{4}}^{\frac{\pi}{4}} d x \sqrt{\frac{B_{1}^{2}(2+\cos 4 x)-K}{2+\cos 4 x}}=0
$$

or:

$$
F\left(B_{1}, B_{2}, K\right)=B_{2}-\Phi\left(B_{1}, K\right)=0
$$

As a function of $\Phi, 1 / v^{2}$ can be written:

$$
\frac{1}{v^{2}}=\lim _{K \rightarrow B_{1}^{2}}\left[\frac{8 \omega \frac{\partial \Phi}{\partial K}}{\alpha-\frac{\partial \Phi}{\partial B_{1}}}\right]_{B_{1}=m_{1} \hbar}
$$

where:

$$
\begin{gathered}
\frac{\partial \Phi}{\partial K}=-\frac{1}{\pi} \int_{-\frac{\pi}{4}}^{\frac{\pi}{4}} d x \frac{1}{\sqrt{(2+\cos 4 x)\left[B_{1}^{2}(2+\cos 4 x)-K\right]}} \\
\frac{\partial \Phi}{\partial B_{1}}=\frac{2}{\pi} \int_{-\frac{\pi}{4}}^{\frac{\pi}{4}} d x \sqrt{\frac{B_{1}^{2}(2+\cos 4 x)}{B_{1}^{2}(2+\cos 4 x)-K}} .
\end{gathered}
$$

A similar procedure has been used for a more schematic model in [20]. The result is:

$$
\frac{1}{v^{2}}=\frac{16 \omega}{m_{1} \hbar}
$$

where $m_{1} \hbar \simeq E$ (the energy of the system) and $\omega=\left(2 v^{2} g^{2}\right)^{\frac{1}{2}}$. Therefore the chaos-order transition depends by the parameter $\lambda=v^{3} g / E$ : if $0<\lambda<$ $\sqrt{2} / 32$ a relevant region of the phase-space is chaotic, but if $\lambda>\sqrt{2} / 32$ 
the system becomes regular. This result shows that the value of the Higgs field in the vacuum $v$ plays an important role: for large values makes the system regular, in agreement with previous numerical calculations [3]. Also the Yang-Mills coupling constant $g$ has the same role. Instead if $v$ and $g$ are fixed there is an order-chaos transition increasing the energy $E$.

In conclusion, we have shown for the spatially homogenous $\mathrm{SU}(2) \mathrm{YMH}$ system that the quantum resonance criterion, which describes the onset of widespread chaos associated to semiclassical crossing between separatices of different quantum multiplets, gives an analytical estimation of the classical chaos-order transition as a function of the higgs vacuum, the Yang-Mills coupling constant and the energy of the system.

We observe that a classical chaos-order transition has been found numerically for the monopole solution [4] and the sphaleron solution [5] of the SU(2) YMH theory. In the future, it will be of great importance to find analytical estimations of the onset of chaos also for these more realistic solutions.

$$
* * * * *
$$

The author is greatly indebted to Profs. G. Benettin, S. Graffi, V. R. Manfredi and Dr. A. Vicini for many enlightening discussions. The author acknowledges Prof. J. M. G. Gomez for his kindly hospitality at the Department of Atomic, Molecular and Nuclear Physics of "Complutense" University, and the "Ing. Aldo Gini" Foundation for a partial support. 


\section{References}

[1] G. K. Savvidy: Phys. Lett. B 130, 303 (1983)

[2] G. K. Savvidy: Phys. Lett. B 159, 325 (1985)

[3] G. K. Savvidy: Nucl. Phys. B 246, 302 (1984)

[4] T. Kawabe, S. Ohta: Phys. Rev. D 44, 1274 (1991)

[5] T. Kawabe, S. Ohta: Phys. Lett. B 334, 127 (1994)

[6] T. Kawabe: Phys. Lett. B 343, 254 (1995)

[7] M. S. Sriram, C. Mukku, S. Lakshmibala, B. A. Bambah: Phys. Rev.

D 49, 4246 (1994)

[8] A. J. Lichtenberg, M. A. Lieberman: Regular and Stochastic Motion (Springer-Verlag, 1983)

[9] H. Poincarè: New Methods of Celestial Mechanics, vol. 3, ch. 27 (Transl. NASA Washington DC 1967); M. Henon: Physica D 5, 412 (1982)

[10] M. Toda: Phys. Lett. A 48, 335 (1974)

[11] G. Benettin, R. Brambilla, L. Galgani: Physica A 87, 381 (1977)

[12] S. Graffi, T. Paul, H. J. Silverstone: Phys. Rev. Lett. 59, 255 (1987)

[13] B. V. Chirikov: Phys. Rep. 52, 263 (1979)

[14] C. Itzykson, J. B. Zuber: Quantum Field Theory (McGraw-Hill, 1985)

[15] M. Born: Mechanics of the Atom (Bell, 1960); J. Bartels and S. J. Chang: Phys. Rev. A 41, 598 (1990)

[16] M. C. Gutzwiller: Chaos in Classical and Quantum Mechanics (SpringerVerlag, 1991) 
[17] D. Delande: Chaos and Quantum Physics, in Les Houches Summer School 1989, Ed. M. J. Giannoni, A. Voros and J. Zinn-Justin (Elsevier Science Publishing, 1989)

[18] V. P. Maslov and M. V. Fedoriuk: Semi-Classical Approximation in Quantum Mechanics (Reidel Publishing Company, 1981); W. P. Reinhardt: in The Mathematical Analysis of Physical Systems, Ed. R. E. Mickens and R. Van Nostrand (1984)

[19] S. Graffi, V. R. Manfredi, L. Salasnich: Nuovo Cim. B 109, 1147 (1994)

[20] S. Graffi, V. R. Manfredi, L. Salasnich: Mod. Phys. Lett. B 7, 747 $(1995)$ 